\title{
Three-point vertices in Landau-gauge Yang-Mills theory
}

\author{
Attilio Cucchieri, ${ }^{1, *}$ Axel Maas, ${ }^{2,3,+}$ and Tereza Mendes ${ }^{1,4,+}$ \\ ${ }^{1}$ Instituto de Física de São Carlos, Universidade de São Paulo, Caixa Postal 369, 13560-970 São Carlos, SP, Brazil \\ ${ }^{2}$ Department of Complex Physical Systems, Institute of Physics, Slovak Academy of Sciences, Dúbravská cesta 9 , \\ SK-845 11 Bratislava, Slovakia \\ ${ }^{3}$ Department of Theoretical Physics, Institute of Physics, Karl-Franzens University Graz, Universitätsplatz, 5, A-8010 Graz, Austria \\ ${ }^{4}$ DESY-Zeuthen, Platanenallee 6, 15738 Zeuthen, Germany
}

(Received 13 March 2008; published 28 May 2008)

\begin{abstract}
Vertices are of central importance for constructing QCD bound states out of the individual constituents of the theory, i.e. quarks and gluons. In particular, the determination of three-point vertices is crucial in nonperturbative investigations of QCD. We use numerical simulations of lattice gauge theory to obtain results for the 3-point vertices in Landau-gauge SU(2) Yang-Mills theory in three and four space-time dimensions for various kinematic configurations. In all cases considered, the ghost-gluon vertex is found to be essentially tree-level-like, while the three-gluon vertex is suppressed at intermediate momenta. For the smallest physical momenta, reachable only in three dimensions, we find that some of the three-gluonvertex tensor structures change sign.
\end{abstract}

DOI: 10.1103/PhysRevD.77.094510

PACS numbers: 11.15.Ha, 12.38.Aw, 14.70.Dj

\section{INTRODUCTION}

Vertices describe the basic interactions between the elementary degrees of freedom of QCD and are thus of central importance for the understanding of nontrivial phenomena in the physics of strong interactions. The properties of vertices, in particular, in the momentum regime of the average constituent momentum, are crucial for the formation of bound states. Furthermore, the far-infrared behavior of vertices should be connected to the confining properties of the theory, since confinement necessarily originates in the interaction of the fields. Quite clearly, a determination of these vertices is an important step in any understanding of the nonperturbative regime of QCD. Finally, in QCD the vertices are also important for the breaking of chiral symmetry, and thus are a central ingredient in the understanding of hadron physics.

In general, the vertices are gauge-dependent quantities. This means that one must understand how gauge-invariant objects, such as hadrons, are constructed from gaugevariant objects, i.e. quarks and gluons. This question has been widely studied by analytical methods, using, in particular, Dyson-Schwinger equations [1-3]. Most of these studies were carried out either in Landau or in Coulomb gauge and specific models were used for the vertices. Here we consider the (minimal) Landau gauge in order to add results for these vertices from lattice gauge theory. We concentrate only on the vertices in SU(2) Yang-Mills theory. A thorough study of the SU(3) case, as well as of the quark-gluon vertex [4-6], is the next logical step. Let us stress, however, that recent lattice studies (in the quenched

\footnotetext{
*attilio@ifsc.usp.br

+axel.maas@uni-graz.at

mendes@ifsc.usp.br
}

case) [7] have provided support to the analytic prediction [1] of identical infrared behavior for Landau-gauge gluon and ghost propagators in $\mathrm{SU}(2)$ and $\mathrm{SU}(3)$ gauge theory in any dimension.

We note that predictions for the infrared behavior of all Green's functions in Landau gauge have been made in four $[4,8-12]$ and also in lower dimensions [13]. These predictions are claimed to be unique under certain assumptions [14]. Furthermore, they yield an infrared enhanced ghost propagator which is accompanied by an infrared finite or vanishing gluon propagator. In particular, an infrared treelevel-like ghost-gluon vertex leads to an infrared vanishing gluon propagator [8].

The analytic results have been extensively tested in lattice gauge theory studies [15-20] and were confirmed in two dimensions [21]. In higher dimensions a major obstacle are finite-volume effects [15,19,21-24]. Nevertheless, it has been recently shown [25] that one can control the infinite-volume extrapolation of the data for the gluon propagator $D(p)$ by considering rigorous lower and upper bounds, expressed in terms of the momentum-space gluon field. As a result, it was found that the Landau-gauge gluon propagator at zero momentum $D(0)$ is finite and nonzero in $3 \mathrm{D}$ and in $4 \mathrm{D}$, while $D(0)=0$ in $2 \mathrm{D}$, in agreement with Ref. [21]. At the same time, the infrared enhancement of the ghost propagator seems to disappear when large lattice volumes are considered [16,24]. However, in Refs. [26] it has been claimed that the analysis of Gribov-Singer-copies effects [27] may modify these results, even though these effects seem to diminish, albeit slowly, with increasing volume. In any case, a better understanding might be obtained by considering upper and lower bounds also for the ghost propagator [28].

Let us remark that in Ref. [29] it was shown that one can also obtain a finite $D(0)$ gluon propagator and a tree-level- 
like ghost propagator at small momenta using the GribovZwanziger approach. Similar results, using numerical solutions of the Dyson-Schwinger equations, have been claimed in [30]. ${ }^{1}$

Here we consider the simplest vertices, i.e. the threepoint vertices. Two of these exist in Landau gauge: the ghost-gluon vertex and the three-gluon vertex. We present results in four dimensions in Sec. II. As we will show, we find an essentially tree-level-like ghost-gluon vertex and a three-gluon vertex that is suppressed at intermediate and small momenta. We note, however, that in four dimensions most of our lattice volumes are rather small and the statistic for the largest volume is low, i.e. for the three-gluon vertex we cannot really probe the asymptotic infrared limit. Therefore, we also present results in three dimensions, in Sec. III. In this case we find a qualitative change for the three-gluon vertex. More precisely, this vertex shows a sign change and an enhancement (in absolute value) in the farinfrared regime.

Technical details of the lattice calculations are given in Appendix A. In particular, the lattice parameters employed are reported in Table I. For the sake of completeness, the data for the propagators are presented in Appendix B.

This work extends previous studies in three and in four dimensions [17,18,32,33] including additional kinematic configurations and larger lattice volumes. Preliminary results have been reported in [34].

\section{VERTICES IN FOUR DIMENSIONS}

In lattice calculations, one can easily evaluate the full Green's functions, while the vertex functions cannot be computed directly. Let us recall that the ghost-gluon vertex has only one (color-antisymmetric) tensor structure in its full Green's function. On the contrary, the three-gluon vertex has a much richer structure. It is possible to determine the contribution of each tensor structure by considering the appropriate projection of the full Green's function. Here only one such tensor structure will be investigated, the one given by the projection ${ }^{2}$

$$
G=\frac{\Gamma_{a b c}^{\mathrm{t}} D_{a d} D_{b e} D_{c f} \Gamma_{d e f}}{\Gamma_{a b c}^{\mathrm{tl}} D_{a d} D_{b e} D_{c f} \Gamma_{d e f}^{\mathrm{t}}},
$$

where $\Gamma$ denotes the vertices, $D$ are (gluon or ghost) propagators and the indices are multi-indices encompassing field-type, Lorentz and color indices. The superscript tl indicates tree-level quantities. Note that this quantity is dimensionless.

Clearly, for the ghost-gluon vertex the above tensor structure (1) reduces to the single tensor structure characterizing its full Green's function. On the contrary, in the

\footnotetext{
${ }^{1}$ However, see the remarks in [3] on these solutions.

${ }^{2}$ The collinear singularities discussed in [11] are not affecting the tensor structure considered here. We are grateful to Markus Huber and Kai Schwenzer for providing this information.
}

case of the three-gluon vertex, it is a linear combination of the transverse vertex tensor structures in the conventional separation scheme discussed in Ref. [35]. Note that the normalization in (1) is chosen so as to absorb trivial kinematic factors, yielding $G$ equal to 1 if the full and the treelevel vertices coincide. A more detailed discussion of the quantity (1) can be found in [18].

One should also recall that three-point vertices depend on two independent external momenta. Using translational and rotational invariance, this dependence can be reduced to three kinematic quantities. These will be chosen as the magnitude of two of the external momenta and the angle between them. In the case of the three-gluon vertex, due to bosonic symmetry, it is irrelevant which of the momenta of the three external lines are chosen as independent. For the ghost-gluon vertex, we consider the gluon and the ghost momenta. Note that, since Landau gauge is ghost-antighost symmetric [1], the ghost and antighost momenta can be exchanged without modifying the result.

Of course, in lattice calculations, the kinematic variables have to be compatible with the (hyper-cubic) symmetry of the lattice. As discussed in Ref. [18], we consider two specific kinematic configurations, denoted, respectively, as orthogonal and equal. In the first case the two external momenta are chosen orthogonal to each other, i.e. the angle in between is equal to 90 degrees. In the equal case the two momenta have equal magnitude and the angle is 60 degrees. The first case allows one to reach the smallest possible nonzero momentum on a given lattice. The second case reduces the problem to a one-scale problem, which is attractive in studies using functional methods in the farinfrared $[4,10,13,14]$. Indeed, in that case it is predicted that all $n$-point vertices behave as power laws in this single external scale $[4,10,14]$. In addition, if the exponent of the power law of one of the vertices is known, the others are all fixed [14].

The remaining technical details for the determination of vertices can be found in [18] and in Appendix A. A list of the studied systems is given in Table I in Appendix A.

Our results for the ghost-gluon vertex $G^{c \bar{c} A}$ are shown in Fig. 1. For all momentum configurations, the vertex is essentially flat, except for a shallow maximum at about $1 \mathrm{GeV}$. Therefore, the ghost-gluon vertex is essentially unmodified compared to its tree-level version. In particular, this is the case both for the vertex measured in the equal (symmetric) or in the orthogonal momentum configuration. This result is in agreement with previous studies in 4D [17] and with data obtained in lower dimensional systems (see Sec. III and Refs. [18,21]). Furthermore, it is in agreement with results from Dyson-Schwinger equation calculations [36]. As noted above, this result can also be used as an input to solve the complete tower of functional equations for the Yang-Mills Green's functions.

The results for the three-gluon vertex $G^{A^{3}}$ are shown in Fig. 2. As has been previously observed [34], the vertex is 

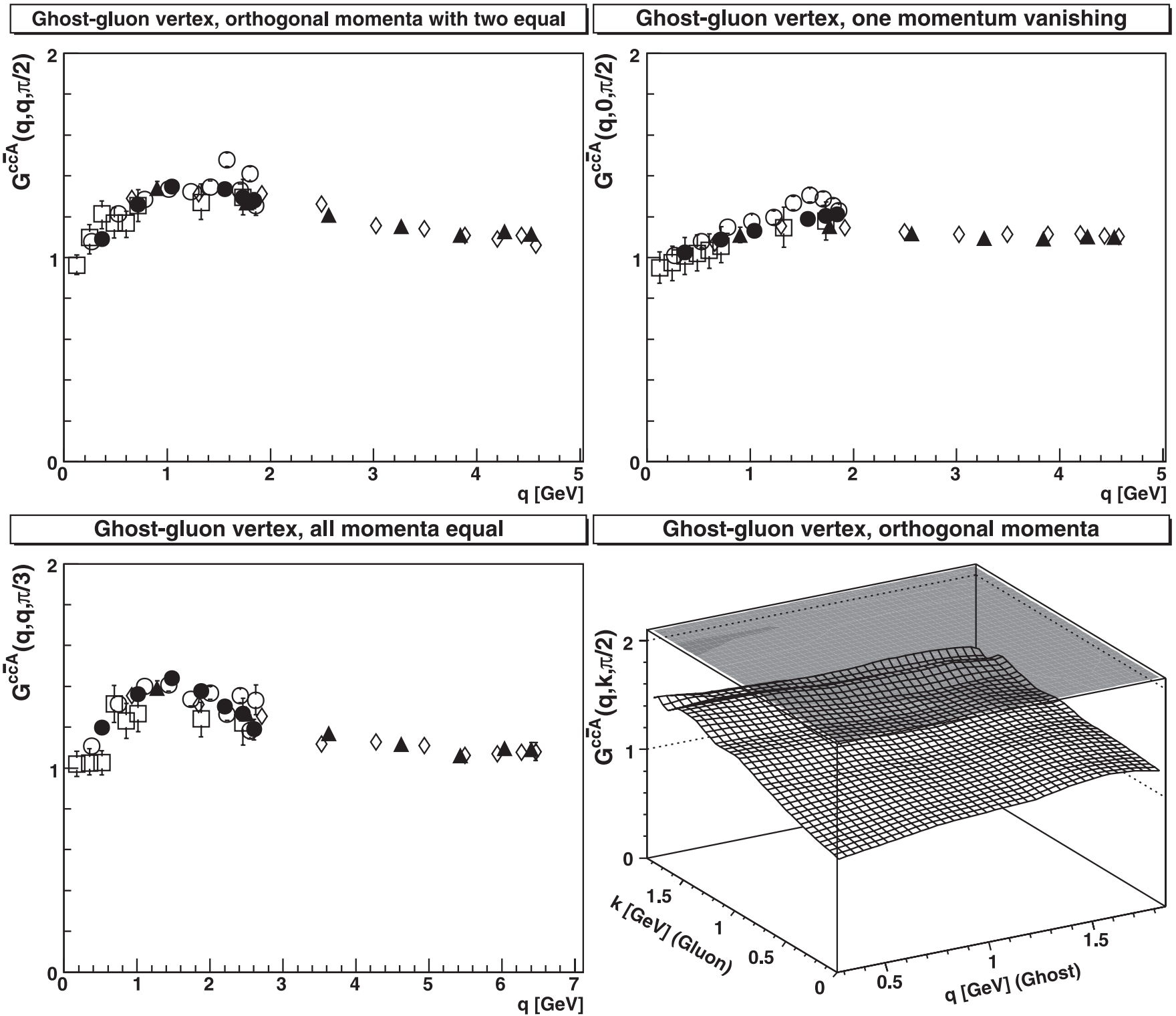

Ghost-gluon vertex, orthogonal momenta

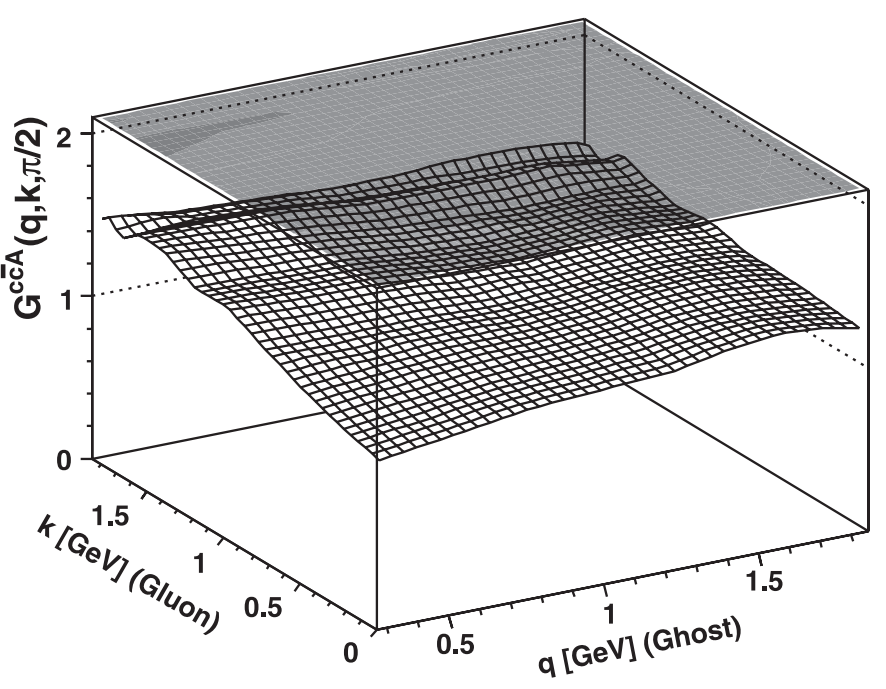

FIG. 1. The ghost-gluon vertex in four dimensions. The bottom-left panel shows the so-called equal configuration. The bottom-right panel shows the orthogonal configuration. The top panels give cuts through the orthogonal plane. The top-left panel shows the case of ghost and gluon momentum with equal magnitude. The top-right panel shows the case with a vanishing gluon momentum. Full triangles are from a $16^{4}$ lattice at $\beta=2.5$, open diamonds from a $22^{4}$ lattice at $\beta=2.5$, full circles from a $16^{4}$ lattice at $\beta=2.2$, open circles from a $22^{4}$ lattice at $\beta=2.2$ and open squares from a $48^{4}$ lattice at $\beta=2.2$. The bottom-right panel shows the data only for the $22^{4}$ lattice at $\beta=2.2$. All data are in physical units.

suppressed at midmomentum compared to a bare one, independently of the momentum configuration considered. However, even on the largest lattice it is not clear whether (or not) the vertex becomes negative at small momenta, or shows a divergence towards zero momentum. Indication of such a divergence was found in lower dimensions [18,21]. Note that, as in lower dimensions [18], there is also a clear increase in the statistical noise for large lattice momenta. A possible solution to this problem, in order to obtain a good signal-to-noise ratio also at large physical momenta, would be of course to simulate at larger values of $\beta$.
Unfortunately, the three-gluon vertex is in general very noisy, and thus the results for the expensive case of the $48^{4}$ lattice are affected by very strong statistical fluctuations. Nonetheless, the data points with acceptable errors confirm the trend seen on the smaller lattices.

As said above, it is difficult to compare the result for the three-gluon vertex $G^{A^{3}}$ with results obtained using functional methods, since one needs a sufficiently large statistic for large lattice volumes, in order to probe the true infrared limit. However, the midmomentum suppression observed may already be relevant for phenomenological applications 

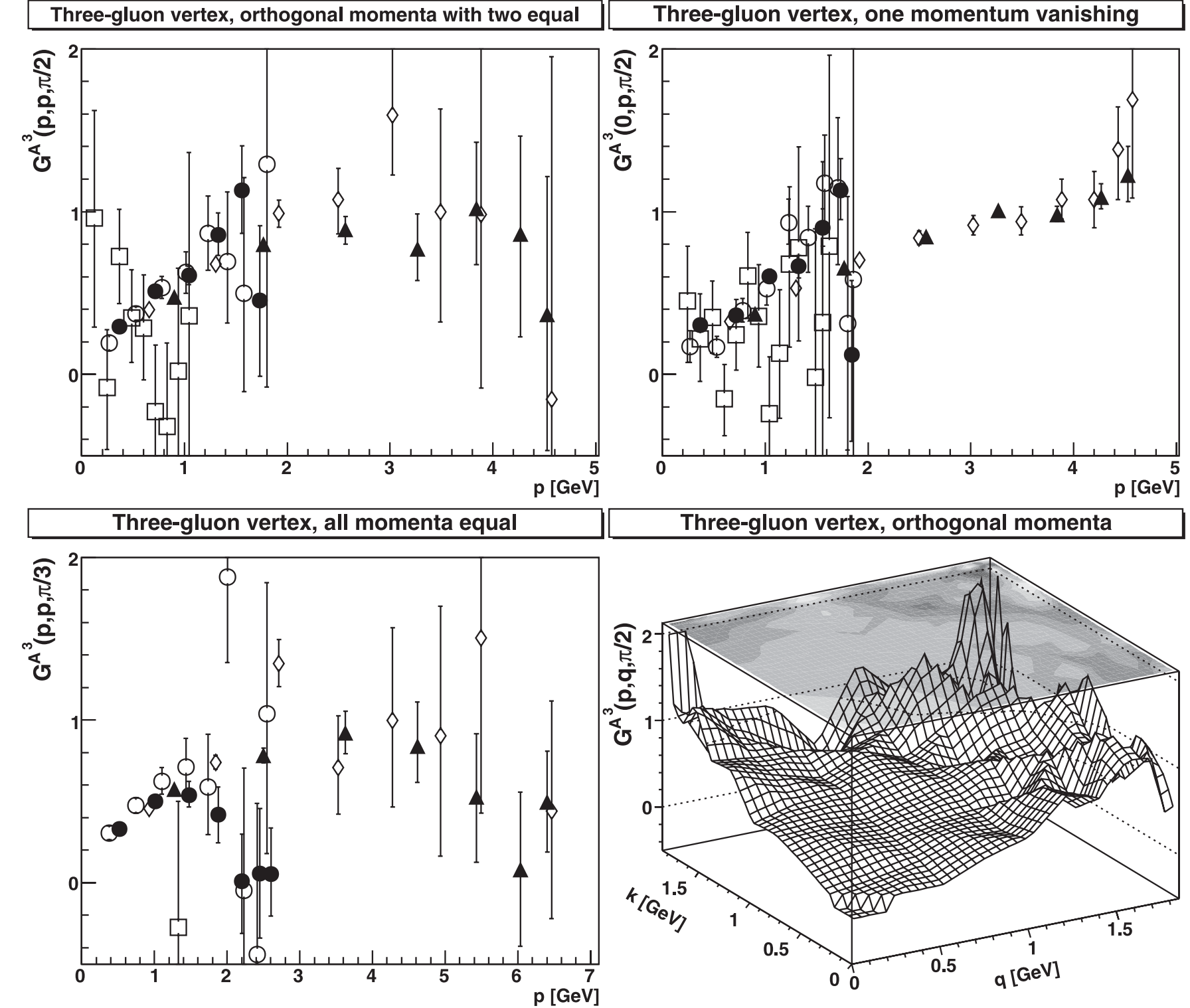

FIG. 2. Same as in Fig. 1 but the data now refer to the three-gluon vertex in four dimensions. Note that, due to the relatively low statistics in the $48^{4}$ case, plot points with an absolute error large than 1.5 have been dropped for this system.

and for the determination of the quark-gluon vertex using functional methods [5]. This suppression is also certainly useful in understanding the discrepancies obtained for the propagators when comparing results from functional methods to lattice studies $[1,3]$.

\section{VERTICES IN THREE DIMENSIONS}

For the numerical determination of the vertices in three dimensions we follow the same procedure used in the fourdimensional case. In particular, we consider again the contraction defined in Eq. (1) above.

The results for the ghost-gluon vertex, shown in Fig. 3, are found to be qualitatively similar to those obtained in 4D (see Sec. II above and Ref. [17]). They are also in agree- ment with the results in 2D [21] and with results obtained in 3D for smaller physical volumes [18]. In particular, the vertex is essentially flat and constant in the infrared limitexcept for a small maximum at midmomenta. As in four dimensions, this result coincides with predictions obtained using functional methods [36]. Note that the maximum of the vertex occurs in the range $[0.5,1] \mathrm{GeV}$, i.e. at slightly larger momenta than the maximum in the gluon propagator [19], and that the position of this maximum agrees very well with analytic predictions [36].

The results for the three-gluon vertex are shown in Fig. 4. Again, the vertex is in qualitative agreement with results obtained in higher [34] and in lower dimensions [21]. In particular, it becomes negative at essentially the 

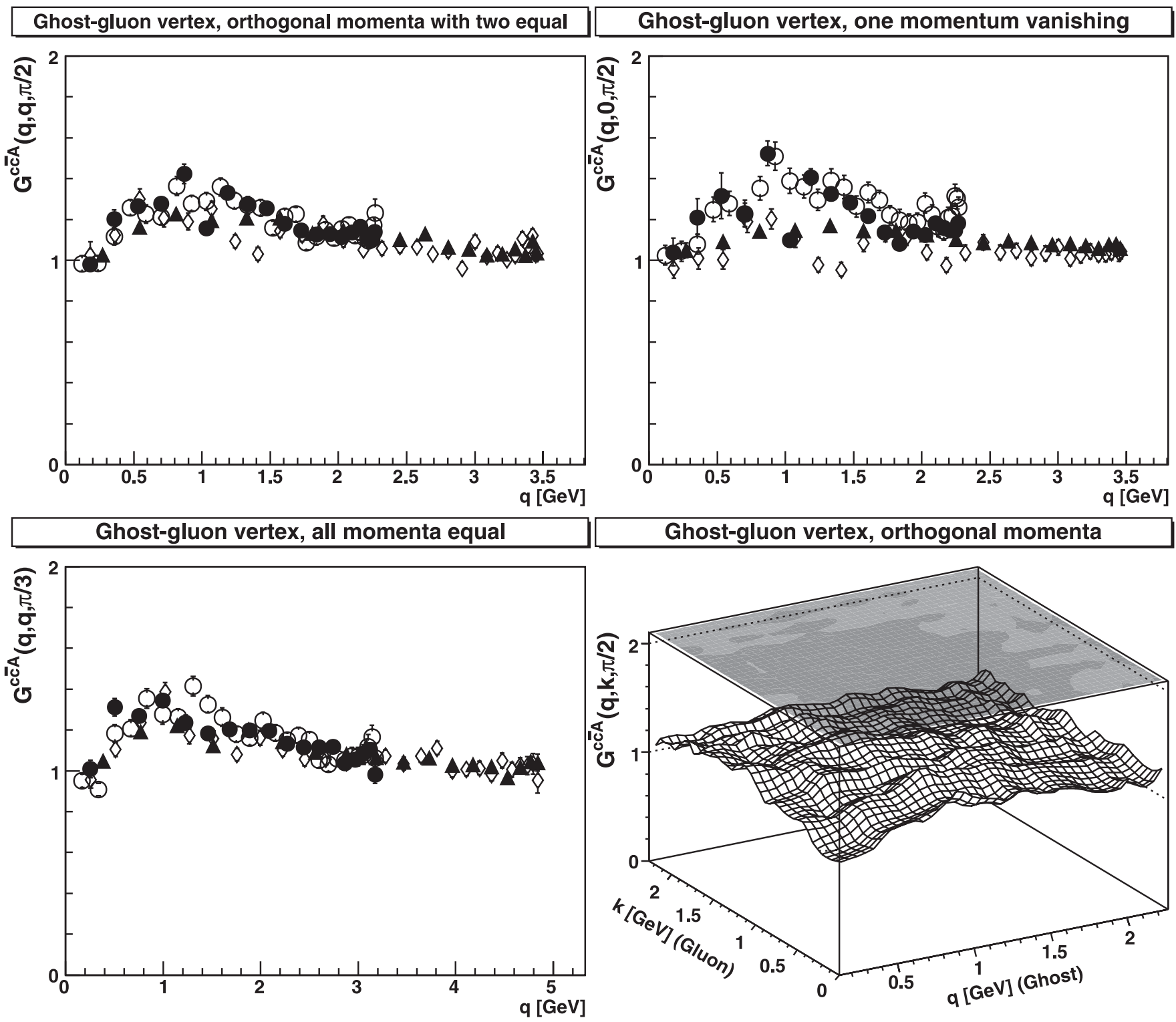

FIG. 3. The ghost-gluon vertex in three dimensions. The bottom-left panel shows the so-called equal configuration. The bottom-right panel shows the orthogonal configuration. The top panels give cuts through the orthogonal plane. The top-left panel shows the case of ghost and gluon momentum with equal magnitude. The top-right panel shows the case with a vanishing gluon momentum. Full triangles are from a $40^{3}$ lattice at $\beta=6.0$, open diamonds from a $60^{3}$ lattice at $\beta=6.0$, full circles from a $40^{3}$ lattice at $\beta=4.2$ and open circles from a $60^{3}$ lattice at $\beta=4$. . The bottom-right panel shows the data only for the $60^{3}$ lattice at $\beta=4.2$. All data are in physical units.

same momentum where the maximum for the gluon propagator occurs. At even smaller momenta, the quantity $G^{A^{3}}$ becomes rapidly large and negative, suggesting a divergence, as in two dimensions [21]. As a consequence, at least one of the two tensor structures contributing to $G^{A^{3}}$ should be infrared divergent (with a negative prefactor). Of course, if they both diverge, then (at least) the term with the stronger divergence should have a negative prefactor. Let us recall that, in the case of one vanishing gluon momentum, only one of the two tensor components (the one proportional to the tree-level component) contributes to the three-gluon vertex [33]. Thus, in this case, the vertex self-energy should be negative and larger than the treelevel result. Finally, let us note that a divergence for the three-gluon vertex is predicted by functional methods $[10,13,37]$, although the sign of the prefactor is either not accessible [10,13] or it is positive [37]. Since the sign of this prefactor depends on the interplay between various contributions, this discrepancy will probably not be easily resolved. 


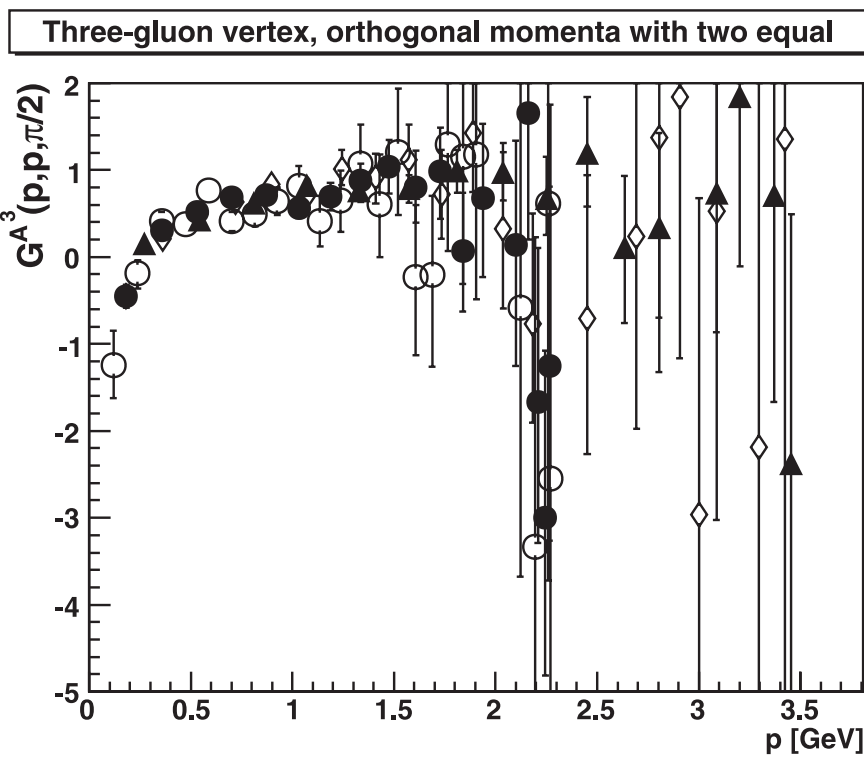

Three-gluon vertex, one momentum vanishing
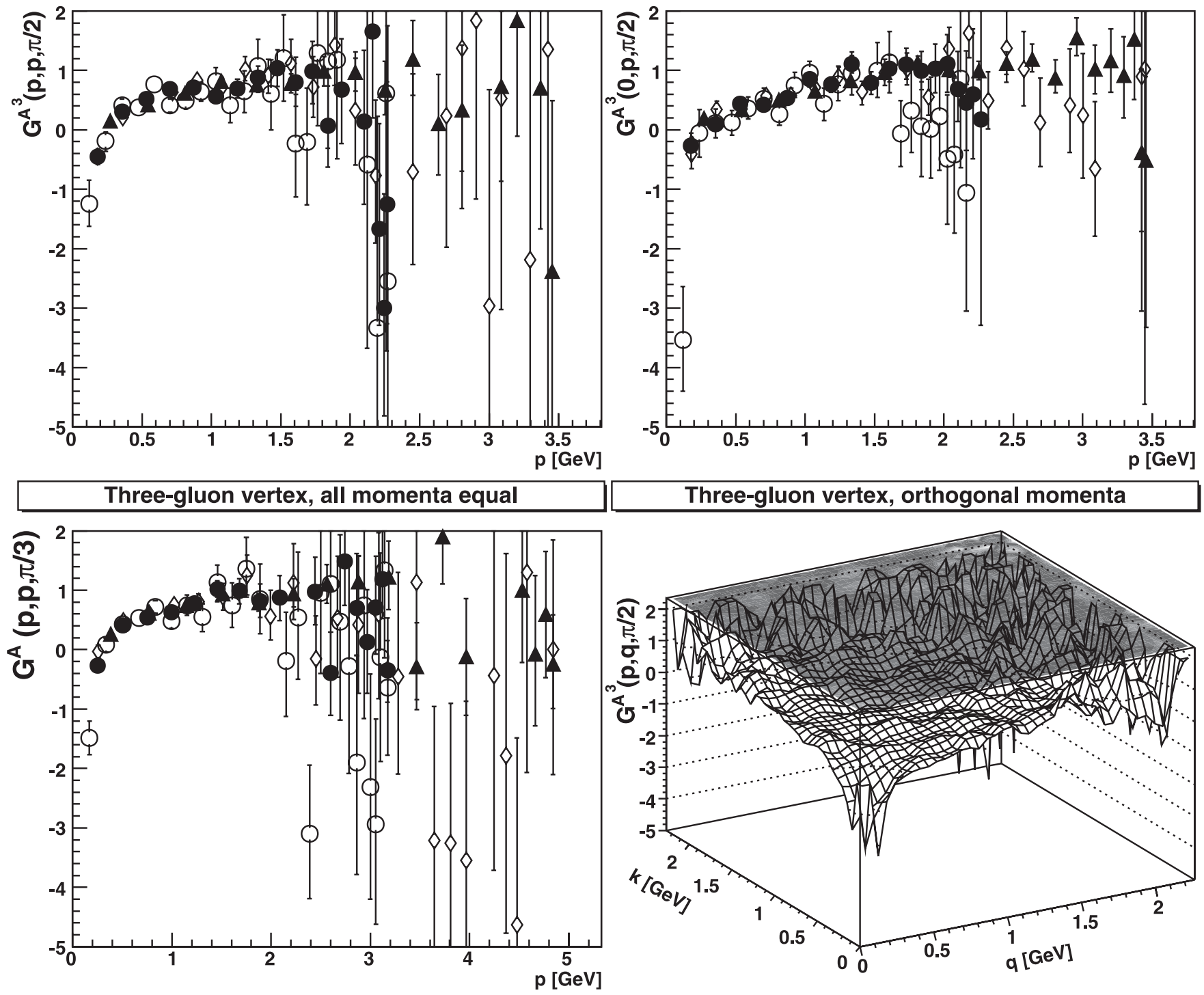

Three-gluon vertex, orthogonal momenta

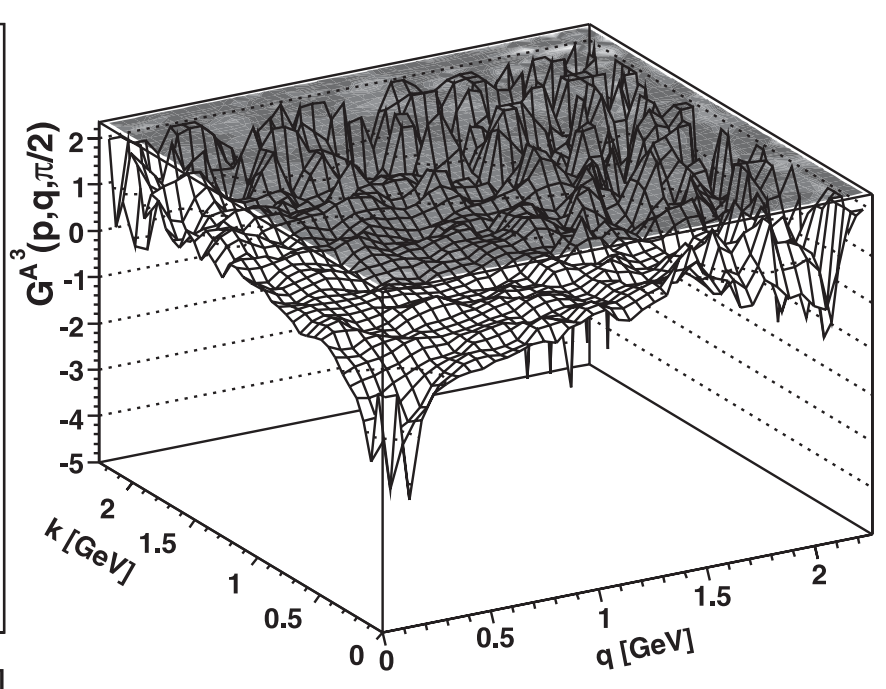

FIG. 4. Same as in Fig. 3 but the data now refer to the three-gluon vertex in three dimensions.

\section{SUMMARY}

As shown above, we find that the ghost-gluon vertex is essentially constant for all momenta in three and four dimensions. At the same time, the three-gluon vertex is found to be suppressed at midmomentum as well as at the smallest momentum reachable in four dimensions. In three dimensions, a clear zero-crossing with a likely infrared divergence is observed.

Combining these results with previous data in four [17], three [18] and in two dimensions [21], it is suggestive that, for any number of dimensions $d$ and for the momentum configurations considered, the ghost-gluon vertex is infrared constant and nonzero, while the three-gluon vertex is (negative) infrared divergent. These results are in good agreement with predictions and assumptions in functional calculations [10,13,36,37].

Let us also note that, in the case of the threegluon vertex, the midmomentum behavior is quite different from the tree-level one. The consequences of this result for the quark-gluon vertex, as well as the relevance for bound-state calculations, are interesting open questions. Finally, it should be remarked that inspecting the various terms in the Dyson-Schwinger equation of the gluon propagator makes it clear that genuine two-loop contributions, usually neglected in such calculations, will likely be important at intermediate momenta. 


\section{ACKNOWLEDGMENTS}

A. M. was supported by the DFG under Grant Nos. MA 3935/1-1 and MA 3935/1-2 and by the FWF under Grant No. P20330. A.C. and T. M. were partially supported by FAPESP (under Grant Nos. 00/05047-5 and 05/59919-7) and by CNPq (including Grant No. 476221/2006-4). T. M. also thanks the Alexander von Humboldt Foundation for financial support. The simulations for the volume $V=48^{4}$ have been done on the IBM supercomputer at São Paulo University (FAPESP Grant No. 04/08928-3). The ROOT framework [40] has been used in this project.

\section{APPENDIX A: TECHNICALITIES}

The lattice simulations have been performed essentially in the same way as in our previous investigations on smaller lattices [18]. In particular, a standard Wilson action has been used. The parameters of the individual runs can be found in Table I. Gribov-Singer copy effects [27] have not been taken into account. Finally, the calculation of the vertices and the error determination has also been performed as in Ref. [18]. In particular, note that all errors represent a $68 \%$ confidence level.

\section{APPENDIX B: PROPAGATORS}

Since the gluon and ghost propagators are necessary in the process of amputating the Green's functions in order to obtain the vertices, we report here (for completeness) our data for the scalar part of the gluon propagator $D$ and for the ghost propagator $D_{G}$. These data are obtained as described in Ref. [18]. Results are shown in Fig. 5 for four dimensions and in Fig. 6 for three dimensions.

As obtained in previous studies for similar lattice volumes, both in 3D and in 4D one finds an infrared diverging ghost propagator. At the same time, when considering the lattice volume $48^{4}$ at $\beta=2.2$, the gluon propagator seems to display a plateau or to get slightly suppressed at small momenta. On the other hand, there is a distinct maximum in three dimensions for all but the smallest volume. Let us recall that in the context of the Gribov-Zwanziger scenario $[27,38,39]$ studies by Dyson-Schwinger equations predict that all Green's functions behave in the far infrared like power laws $[10,14]$ —at least in the case when all momenta have the same magnitude. These power laws have characteristic infrared exponents in the continuum. Because of finite-volume effects, one expects that the effective infrared exponents obtained from lattice simulations [15,23] should converge to the continuum results when the infinite-volume limit is taken. This is indeed the case in two dimensions [21]. On the other hand, as said in the Introduction, recent results in $3 \mathrm{D}$ and in $4 \mathrm{D}$ using very large lattices $[16,24,25,28]$ show evidence that the gluon propagator is finite (and nonzero) at zero momentum and that the ghost propagator has an infrared exponent very close to zero.

TABLE I. Number of configurations considered in our numerical simulations. The value of the lattice spacing $a$ has been taken from Ref. [19] in three dimensions and from Ref. [31] in four dimensions. Sweeps indicates the number of sweeps between two consecutive gauge-fixed measurements. More details on the generation of the configurations, error-determination, etc. can be found in Ref. [18].

\begin{tabular}{cccccccc}
\hline \hline$d$ & Vertex & $N$ & $\beta$ & $a^{-1}[\mathrm{GeV}]$ & Configurations & Sweeps & $L=V^{1 / d}[\mathrm{fm}]$ \\
\hline 3 & Ghost-gluon & 40 & 6.0 & 1.733 & 1267 & 50 & 4.5 \\
3 & Ghost-gluon & 60 & 6.0 & 1.733 & 460 & 70 & 6.8 \\
3 & Ghost-gluon & 40 & 4.2 & 1.136 & 1077 & 50 & 6.9 \\
3 & Ghost-gluon & 60 & 4.2 & 1.136 & 367 & 70 & 10 \\
3 & Three-gluon & 40 & 6.0 & 1.733 & 9709 & 50 & 4.5 \\
3 & Three-gluon & 60 & 6.0 & 1.733 & 5017 & 70 & 6.8 \\
3 & Three-gluon & 40 & 4.2 & 1.136 & 11095 & 50 & 6.9 \\
3 & Three-gluon & 60 & 4.2 & 1.136 & 8114 & 70 & 10 \\
4 & Ghost-gluon & 16 & 2.5 & 2.309 & 1336 & 30 & 1.4 \\
4 & Ghost-gluon & 22 & 2.5 & 2.309 & 1248 & 50 & 1.9 \\
4 & Ghost-gluon & 16 & 2.2 & 0.938 & 1351 & 30 & 3.4 \\
4 & Ghost-gluon & 22 & 2.2 & 0.938 & 1043 & 50 & 4.7 \\
4 & Ghost-gluon & 48 & 2.2 & 0.938 & 100 & 100 & 10.1 \\
4 & Three-gluon & 16 & 2.5 & 2.309 & 11446 & 30 & 1.4 \\
4 & Three-gluon & 22 & 2.5 & 2.309 & 6291 & 50 & 1.9 \\
4 & Three-gluon & 16 & 2.2 & 0.938 & 8600 & 30 & 3.4 \\
4 & Three-gluon & 22 & 2.2 & 0.938 & 5365 & 50 & 4.7 \\
4 & Three-gluon & 48 & 2.2 & 0.938 & 3396 & 100 & 10.1 \\
\hline \hline
\end{tabular}




\section{Gluon propagator at low momenta}

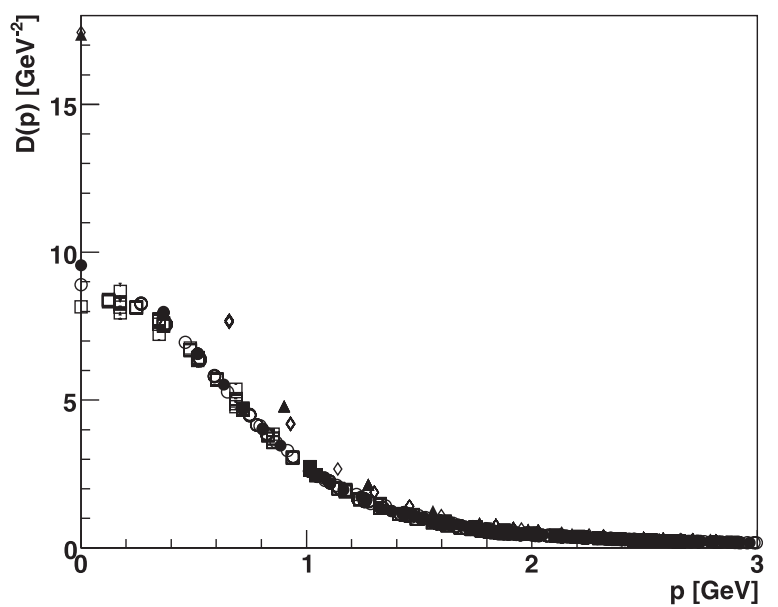

Ghost dressing function

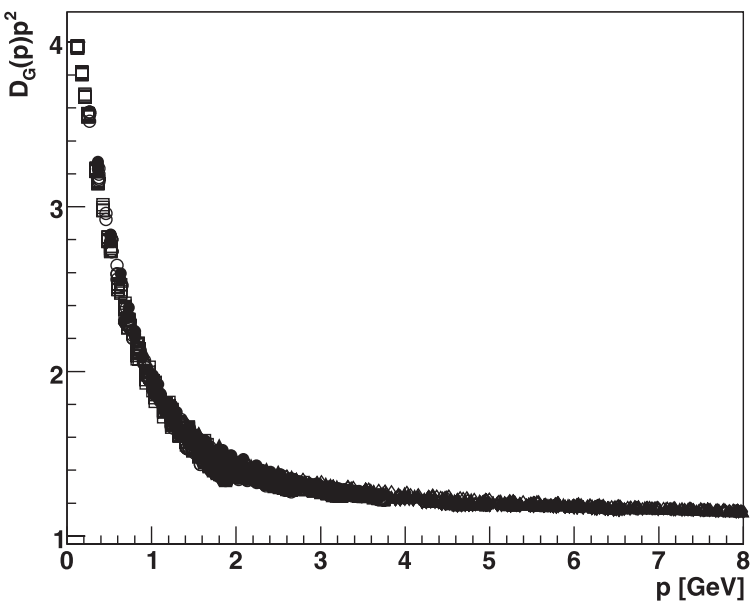

FIG. 5. Here we show the gluon propagator (top panel) and the ghost dressing function (bottom panel), both in four dimensions. Full triangles are from a $16^{4}$ lattice at $\beta=2.5$, open diamonds from a $22^{4}$ lattice at $\beta=2.5$, full circles from a $16^{4}$ lattice at $\beta=2.2$, open circles from a $22^{4}$ lattice at $\beta=2.2$ and open squares from a $48^{4}$ lattice at $\beta=2.2$.

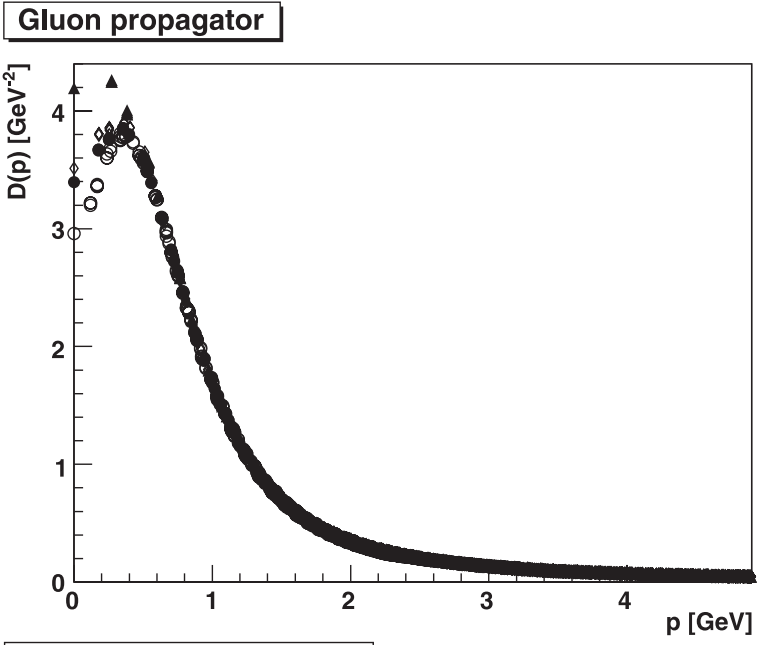

Ghost dressing function

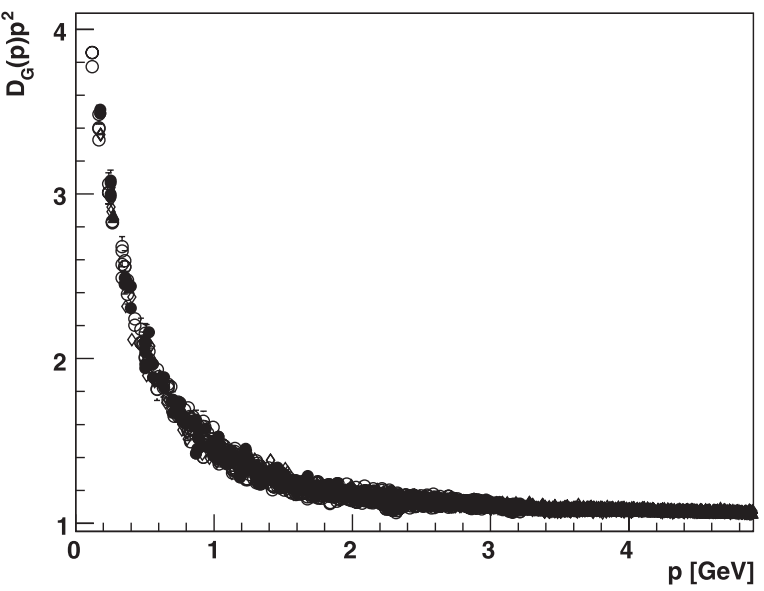

FIG. 6. Same as in Fig. 5 for the three-dimensional case. Full triangles are from a $40^{3}$ lattice at $\beta=6.0$, open diamonds from a $60^{3}$ lattice at $\beta=6.0$, full circles from a $40^{3}$ lattice at $\beta=$ 4.2 and open circles from a $60^{3}$ lattice at $\beta=4.2$.
[1] R. Alkofer and L. von Smekal, Phys. Rep. 353, 281 (2001).

[2] P. Maris and C. D. Roberts, Int. J. Mod. Phys. E 12, 297 (2003); A. Holl, C. D. Roberts, and S. V. Wright, arXiv: nucl-th/0601071.

[3] C. S. Fischer, J. Phys. G 32, R253 (2006).

[4] R. Alkofer, C. S. Fischer, and F. J. Llanes-Estrada, Mod. Phys. Lett. A (to be published).

[5] F. J. Llanes-Estrada, C. S. Fischer, and R. Alkofer, Nucl. Phys. B, Proc. Suppl. 152, 43 (2006).
[6] J. Skullerud and A. Kizilersu, J. High Energy Phys. 09 (2002) 013; J. I. Skullerud, P. O. Bowman, A. Kizilersu, D. B. Leinweber, and A. G. Williams, J. High Energy Phys. 04 (2003) 047; J. I. Skullerud, A. Kizilersu, P. O. Bowman, D. B. Leinweber, and A. G. Williams, Nucl. Phys. B, Proc. Suppl. 128, 117 (2004); J. I. Skullerud, P. O. Bowman, A. Kizilersu, D. B. Leinweber, and A. G. Williams, Nucl. Phys. B, Proc. Suppl. 141, 244 (2005); A. Kizilersu, D. B. Leinweber, J. I. Skullerud, and A. G. Williams, Eur. Phys. J. C 50, 871 (2007). 
[7] A. Cucchieri, T. Mendes, O. Oliveira, and P. J. Silva, Phys. Rev. D 76, 114507 (2007); A. Sternbeck, L. von Smekal, D. B. Leinweber, and A. G. Williams, Proc. Sci., LAT2007 (2007) 340 [arXiv:hep-lat/0710.1982]; A. Maas and S. Olejnik, J. High Energy Phys. 02 (2008) 070.

[8] C. Lerche and L. von Smekal, Phys. Rev. D 65, 125006 (2002).

[9] J. M. Pawlowski, D. F. Litim, S. Nedelko, and L. von Smekal, Phys. Rev. Lett. 93, 152002 (2004).

[10] R. Alkofer, C. S. Fischer, and F. J. Llanes-Estrada, Phys. Lett. B 611, 279 (2005).

[11] R. Alkofer, M.Q. Huber, and K. Schwenzer, arXiv:0801.2762.

[12] C. Kellermann and C. S. Fischer, arXiv:0801.2697.

[13] M. Q. Huber, R. Alkofer, C. S. Fischer, and K. Schwenzer, Phys. Lett. B 659, 434 (2008).

[14] C.S. Fischer and J. M. Pawlowski, Phys. Rev. D 75, 025012 (2007).

[15] A. Cucchieri, A. Maas, and T. Mendes, Phys. Rev. D 75, 076003 (2007).

[16] O. Oliveira and P. J. Silva, arXiv:0705.0964.

[17] A. Cucchieri, T. Mendes, and A. Mihara, J. High Energy Phys. 12 (2004) 012; E. M. Ilgenfritz, M. MullerPreussker, A. Sternbeck, and A. Schiller, arXiv: hep-lat/ 0601027.

[18] A. Cucchieri, A. Maas, and T. Mendes, Phys. Rev. D 74, 014503 (2006).

[19] A. Cucchieri, T. Mendes, and A. R. Taurines, Phys. Rev. D 67, 091502 (2003).

[20] J. C. R. Bloch, A. Cucchieri, K. Langfeld, and T. Mendes, Nucl. Phys. B687, 76 (2004); P. O. Bowman, U. M. Heller, D. B. Leinweber, M.B. Parappilly, and A.G. Williams, Phys. Rev. D 70, 034509 (2004); A. Sternbeck, E. M. Ilgenfritz, M. Mueller-Preussker, and A. Schiller, Phys. Rev. D 72, 014507 (2005); A. Sternbeck, E. M. Ilgenfritz, M. Muller-Preussker, A. Schiller, and I. L. Bogolubsky, Proc. Sci., LAT2006 (2006) 076; W. Kamleh, P. O. Bowman, D. B. Leinweber, A. G. Williams, and J. Zhang, Phys. Rev. D 76, 094501 (2007); Ph. Boucaud et al., Eur. Phys. J. A 31, 750 (2007); J. High Energy Phys. 03 (2007) 076.

[21] A. Maas, Phys. Rev. D 75, 116004 (2007).

[22] A. Cucchieri and T. Mendes, Braz. J. Phys. 37, 484 (2007).
[23] C. S. Fischer, A. Maas, J. M. Pawlowski, and L. von Smekal, Ann. Phys. (N.Y.) 322, 2916 (2007).

[24] I. L. Bogolubsky, E. M. Ilgenfritz, M. Muller-Preussker, and A. Sternbeck, Proc. Sci., LAT2007 (2007) 290 [arXiv: hep-lat/0710.1968]; A. Cucchieri and T. Mendes, Proc. Sci., LAT2007 (2007) 297 [arXiv:hep-lat/0710.0412]; A. Sternbeck, L. von Smekal, D. B. Leinweber, and A. G. Williams, Proc. Sci., LAT2007 (2007) 340 [arXiv:hep-lat/ 0710.1982].

[25] A. Cucchieri and T. Mendes, arXiv:0712.3517.

[26] I. L. Bogolubsky, G. Burgio, M. Muller-Preussker, and V. K. Mitrjushkin, Phys. Rev. D 74, 034503 (2006); I. L. Bogolubsky et al., Phys. Rev. D 77, 014504 (2008); 77, 039902(E) (2008).

[27] V. N. Gribov, Nucl. Phys. B139, 1 (1978); I. M. Singer, Commun. Math. Phys. 60, 7 (1978).

[28] A. Cucchieri and T. Mendes, arXiv:hep-lat/0804.2371.

[29] D. Dudal, S.P. Sorella, N. Vandersickel, and H. Verschelde, arXiv:0711.4496.

[30] A. C. Aguilar and A. A. Natale, J. High Energy Phys. 08 (2004) 057; Ph. Boucaud, arXiv:0801.2721; A. C. Aguilar, D. Binosi, and J. Papavassiliou, arXiv:0802.1870.

[31] E. Manousakis and J. Polonyi, Phys. Rev. Lett. 58, 847 (1987); G. S. Bali, J. Fingberg, U. M. Heller, F. Karsch, and K. Schilling, Phys. Rev. Lett. 71, 3059 (1993).

[32] C. Parrinello, Phys. Rev. D 50, R4247 (1994); P. Boucaud, J. P. Leroy, J. Micheli, O. Pene, and C. Roiesnel, J. High Energy Phys. 10 (1998) 017.

[33] B. Alles et al., Nucl. Phys. B502, 325 (1997).

[34] A. Maas, A. Cucchieri, and T. Mendes, Braz. J. Phys. 37N1, 219 (2007).

[35] J. S. Ball and T. W. Chiu, Phys. Rev. D 22, 2550 (1980); 23, 3085(E) (1981).

[36] W. Schleifenbaum, A. Maas, J. Wambach, and R. Alkofer, Phys. Rev. D 72, 014017 (2005).

[37] W. Schleifenbaum, M. Leder, and H. Reinhardt, Phys. Rev. D 73, 125019 (2006).

[38] D. Zwanziger, Phys. Rev. D 69, 016002 (2004).

[39] D. Zwanziger, Phys. Lett. B 257, 168 (1991); Nucl. Phys. B364, 127 (1991); B412, 657 (1994); Phys. Rev. D 65, 094039 (2002); 67, 105001 (2003).

[40] R. Brun and F. Rademakers, Nucl. Instrum. Methods Phys. Res., Sect. A 389, 81 (1997). 